\title{
CORRELACIÓN ENTRE CAPACIDADES COORDINATIVAS Y POTENCIA EN NADADORAS JUVENILES DEL CLUB TIBURONES
}

\author{
CORRELATION BETWEEN COORDINATIVES CAPACITIES AND POWER IN \\ SWIMMERS OF THE TIBURONES CLUB
}

\section{Deissy Johana Porras Suárez}

Licenciada en Educación física, recreación y deporte, Maestrante en Pedagogía de la Cultura Física

Universidad Pedagógica y Tecnológica de Colombia.

E-mail Johana042@hotmail.com

Tel.cel 3134592351

\section{Paulo Jonathan Acosta Tova}

Docente Universidad Pedagógica y Tecnológica de

Colombia-UPTC

Tel. cel 3115802113

E-mail paulo.acosta@uptc.edu.co

acostajon14@hotmail.com

\section{Mónica Yaneth Martínez Martínez}

Docente Universidad Pedagógica y Tecnológica de

Colombia-UPTC

Tel. cel 3143943190

E-mailmonicayaneth.martinez@uptc.edu.co

momikyan@hotmail.com

\section{RESUMEN}

Este estudio tuvo como objetivo determinar la correlación entre capacidades coordinativas y potencia en nadadoras del club Tiburones de la ciudad de Tunja (Colombia). Participaron 15 nadadoras de la categoría juvenil (14 y 15 años). Se evaluaron las capacidades coordinativas con el test motor complejo de Flaviano Lorenzo Caminero y la potencia mediante el test de Bosco, usando plataforma de contacto Axón
Jump 4.0. El análisis estadístico se realizó con el programa SPSS versión 25, la prueba de normalidad se tomó con el Shapiro Wilk y el coeficiente de correlación con la prueba de Pearson, Se analizó la diferencia estadística considerando $p<0.05$, los resultados obtenidos entre la correlación de las capacidades coordinativas y la potencia en cada salto muestran que el $\mathrm{CMJ} r=-0,349$, para el $S J r=-0,245$ y el $A B K r=-0,456$ lo que nos indica que no existe correlación entre ellas, se resalta que de acuerdo a $C M J P=-0,202$; para el $\mathrm{SJ} P=-0,379$ y el despegue en $A B K P=0,087$ fue el más cercano a estar por debajo de 0,05 quiere decir, que es el salto que está más tiene relación con el TMC.

Palabras clave: Capacidades Coordinativas, Potencia Aláctica, nadadoras.

\section{ABSTRACT}

This study was undertaken as a objective determine the correlation between coordinating abilities and power in swimmers of the Tiburones club in the city of Tunja (Colombia). 15 swimmers from the youth category participated (14 and 15 years old). The coordinative capacities were evaluated with the Flaviano Lorenzo Caminero complex test engine and the power through the Bosco test, using the Axon Jump 4.0 contact 
platform. The statistical analysis was performed with the SPSS program version 25, the normality test was taken with the Shapiro Wilk and the correlation coefficient with the Pearson test, the statistical difference was analyzed considering the $p<0.05$, the results obtained between the correlation of the coordinative capacities and the power in each jump show that the CMJ $r=-0.349$, for the $\mathrm{SJ} r$ $=-0.245$ and the ABK $r=-0.456$ which indicates that there is no correlation between them, it is emphasized that according to CMJ $P=-0.202 ;$ for the SJ $P=-0.379$ and the takeoff in $A B K P=0.087$ was the closest to being below 0.05 means that it is the jump that has more relation with the TMC.

Key words: Coordinative Capabilities, Alctic Power, swimmers.

\section{INTRODUCCIÓN}

En natación las capacidades coordinativas juegan un papel importante en el ejecución de la técnica, en donde es indispensable ejecutar movimientos fluidos, constantes, económicos y de calidad, "debido a que un movimiento o posición corporal poco económico en el agua, genera resistencia, disminuye el grado de eficiencia, es decir, bajo aprovechamiento de fuerza aplicada para el desplazamiento" (González, 2009).

(Meinel, 2004) Define las capacidades coordinativas como el desarrollo y la organización de acciones motoras ordenadas hacia un objetivo determinado. Y como la habilidad que tiene el cuerpo o una de sus partes, para desarrollar en secuencia ordenada, armónica y eficaz un gesto o acción determinados.

Identifica siete (7) diferentes capacidades coordinativas. Para estos autores, el constructo capacidades coordinativas se encuentra compuesto por:

Capacidad de Ritmo.

Capacidad de Reacción.

Capacidad de Diferenciación.

Capacidad de Orientación.

Capacidad de Equilibrio

Capacidad de Acoplamiento.

Capacidad de Adaptación.

Esta estructura facilita las mediciones y evaluaciones de la coordinación motriz por la especificidad de cada una de ella. Igual que las capacidades coordinativas.

La potencia es una capacidad importante en la natación ya que con ella se logra ejecutar los movimientos en el menor tiempo posible, implicando un desarrollo de la fuerza y la velocidad traducido en fuerza explosiva, expresada en forma de salto y genera información objetiva que favorece la planificación de gestos motores orientados al incremento del rendimiento muscular de corta duración (Bosco, C., 1982).

\section{MATERIAL Y MÉTODO}

\section{Sujetos}

Participaron 15 nadadoras juveniles , pertenecientes al club Tiburones de la ciudad de Tunja, se realizó una reunión en la piscina municipal con el entrenador del club para solicitar el aval para la ejecución de las pruebas, una vez se explicó el objetivo del mismo, se envió a los padres de familia y/o tutores la información donde se explicaban los objetivos del proyecto, solicitando su consentimiento firmado para la participación en el estudio, garantizando la confidencialidad de la información recogida. 


\section{PROCEDIMIENTO}

Se realizó la evaluación de las capacidades coordinativas mediante el test de Lorenzo Caminero y la potencia con el test de Bosco, mediante la plataforma AXON JUMP 4.0.

Las pruebas tuvieron lugar en la cancha auxiliar de vóleibol de INDEPORTES Boyacá. Todos los participantes cumplieron con los criterios de inclusión de la presente investigación. Para aplicar los diferentes test se realizó la firma de un consentimiento informado de esta manera recibir la autorización del deportista.

Para la ejecución del test de Bosco se utilizó la plataforma Axon Jump 4.0. Para el estudio solo se tomaron en cuenta los siguientes saltos:

- Squat Jump SJ), se inicia en posición erguida con flexión de rodillas de 90 grados, tronco recto y las manos en la cintura, y se realiza un salto vertical, se debe tener en cuenta que en ningún momento durante la ejecución del salto se pueden soltar los brazos, y las rodillas no se deben elevar.

- Counter Movement Jump (CMJ), se inicia en posición erguida sin flexión de rodillas, el tronco recto y los brazos en la cintura, se ejecuta un contra-movimiento hasta llegar a $90^{\circ}$ de flexión de rodilla, para continuar con un salto vertical.

- Abakalov (ABK), similar al CMJ, pero en esta ocasión se permite el uso de brazos para dar impulso, este tipo de salto permite conocer el porcentaje de contribución uso de brazos.

Para medir las capacidades coordinativas se utilizó el test de Lorenzo Caminero, queconsiste en 6 zonas o estaciones ubicadas en un lugar Ilanoyliso de $9 \times 9$ metros. En unazonase valora una capacidad, hasta completar las 6 capacidades coordinativas. Cada sujeto pasa a máxima velocidad por cada zona, hasta completar el circuito. El número de intentospor sujeto fue de tres; el primero de ensayo y dos más cronometrados. Se analizó el tiempo, en segundos, que tardó cada sujeto en completar el circuito (Caminero, 2009).

\section{RESULTADOS}

Los datos fueron analizados utilizando el programa SPSS versión 25, manejando el coeficiente de correlación de Pearson. Se analizó la diferencia estadística considerando un $95 \%$ de confiabilidad y un alfa mayor o igual a 0.05 .

\begin{tabular}{|c|c|c|c|c|}
\hline ID & $\mathbf{A B K}$ & $\mathbf{S J}$ & $\mathbf{C M J}$ & $\mathbf{T M C}$ \\
\hline $\mathbf{1}$ & 21,2 & 19,6 & 20,4 & 29,49 \\
\hline $\mathbf{2}$ & 26,5 & 22 & 20,4 & 28,14 \\
\hline $\mathbf{3}$ & 30,1 & 22,1 & 19,6 & 26,87 \\
\hline $\mathbf{4}$ & 34,2 & 23,8 & 25,5 & 23,82 \\
\hline $\mathbf{5}$ & 21,2 & 26,5 & 22,9 & 27,42 \\
\hline $\mathbf{6}$ & 18,8 & 15,2 & 16,6 & 32,24 \\
\hline $\mathbf{7}$ & 24,6 & 19,6 & 21,12 & 27,24 \\
\hline $\mathbf{8}$ & 28,2 & 24,6 & 25,5 & 30,73 \\
\hline $\mathbf{9}$ & 24,6 & 24,6 & 24,6 & 33,06 \\
\hline $\mathbf{1 0}$ & 27,4 & 25,5 & 22 & 31,59 \\
\hline $\mathbf{1 1}$ & 23,8 & 21,2 & 22 & 29,42 \\
\hline $\mathbf{1 2}$ & 28,2 & 25,5 & 24,6 & 27,16 \\
\hline $\mathbf{1 3}$ & 21,2 & 19,6 & 19,6 & 29,42 \\
\hline $\mathbf{1 4}$ & 26,4 & 26,4 & 26,4 & 27,11 \\
\hline $\mathbf{1 5}$ & 21,2 & 22,9 & 26,4 & 26,57 \\
\hline
\end{tabular}

Tabla 1: Resultados individuales.

En la tabla 1 se observan los resultados individuales de cada uno de los saltos y del TMC, se observa que la mayor altura para el despegue en $A B K$ fue de 34,2 y la menor fue 
de 21,2, para el despegue $\mathrm{SJ}$ el mayor salto fue de 26,5 y el menor de 15,6 , y para CMJ el mayor salto fue de $25,5 \mathrm{y}$ el menor de 16,6 . En cuanto al TMC el menor tiempo fue de $23,82 \mathrm{sg}$ y el mayor tiempo de 33,06sg. Se resalta que la deportista que mas salta en $A B K$, que es el salto coordinativo tiene menos tiempo en el TMC.

\begin{tabular}{|c|c|c|c|c|c|c|}
\hline & \multicolumn{3}{|c|}{ Kolmogorov-Smirnova } & \multicolumn{3}{|c|}{ Shapiro-Wilk } \\
\hline & $\begin{array}{l}\text { Estadísti } \\
\text { co }\end{array}$ & $\mathrm{gl}$ & Sig. & $\begin{array}{c}\text { Estadístic } \\
0\end{array}$ & $\mathrm{gl}$ & Sig. \\
\hline $\begin{array}{l}\text { Peso en } \\
\mathrm{Kg} \text { : }\end{array}$ & 0,100 & $\begin{array}{l}1 \\
5\end{array}$ & $200^{*}$ & 0,955 & $\begin{array}{l}1 \\
5\end{array}$ & $\begin{array}{c}0,60 \\
9\end{array}$ \\
\hline $\begin{array}{l}\text { Talla en } \\
\text { CM: }\end{array}$ & 0,153 & $\begin{array}{l}1 \\
5\end{array}$ & $200^{*}$ & 0,911 & $\begin{array}{l}1 \\
5\end{array}$ & $\begin{array}{c}0,14 \\
3\end{array}$ \\
\hline $\begin{array}{l}\text { Índice de } \\
\text { Masa } \\
\text { Corporal: }\end{array}$ & 0,183 & $\begin{array}{l}1 \\
5\end{array}$ & $\begin{array}{c}0,18 \\
7\end{array}$ & 0,946 & $\begin{array}{l}1 \\
5\end{array}$ & $\begin{array}{c}0,46 \\
8\end{array}$ \\
\hline $\begin{array}{l}\text { Despegue } \\
\text { en ABK: }\end{array}$ & 0,165 & $\begin{array}{l}1 \\
5\end{array}$ & ,200* & 0,954 & $\begin{array}{l}1 \\
5\end{array}$ & $\begin{array}{c}0,59 \\
5\end{array}$ \\
\hline $\begin{array}{l}\text { Despegue } \\
\text { en SJ: }\end{array}$ & 0,136 & $\begin{array}{l}1 \\
5\end{array}$ & $200^{*}$ & 0,928 & $\begin{array}{l}1 \\
5\end{array}$ & $\begin{array}{c}0,25 \\
3\end{array}$ \\
\hline $\begin{array}{l}\text { Despegue } \\
\text { en CMJ: }\end{array}$ & 0,162 & $\begin{array}{l}1 \\
5\end{array}$ & $200^{*}$ & 0,941 & $\begin{array}{l}1 \\
5\end{array}$ & $\begin{array}{c}0,39 \\
8\end{array}$ \\
\hline $\begin{array}{l}\text { Test Motor } \\
\text { complejo } \\
\text { de } \\
\text { Caminero: }\end{array}$ & 0,161 & $\begin{array}{l}1 \\
5\end{array}$ & $200^{*}$ & 0,956 & $\begin{array}{l}1 \\
5\end{array}$ & $\begin{array}{c}0,61 \\
8\end{array}$ \\
\hline $\begin{array}{l}\text { Esto es } \\
\text { a. }\end{array}$ & $\begin{array}{l}\text { ite in } \\
\text { eccic }\end{array}$ & or d & ica & ación verd & & \\
\hline
\end{tabular}

Tabla 2. Prueba de Normalidad

Debido a que se cumplieron los supuestos de normalidad y se buscó hacer una correlación, se procedió a aplicar la prueba de Pearson.

En la tabla 3, se observa la correlación de las capacidades coordinativas y la potencia en cada salto muestran que el $\mathrm{CMJ} r=-0,349$, para el SJ $r=-0,245$ y el $A B K r=-0,456$ lo que nos indica que no existe correlación entre ellas, se resalta que de acuerdo a $\mathrm{CMJ} P=-0,202$; para el $S J P=-0,379$ y el despegue en $A B K P=0,087$ fue el más cercano a estar por debajo de 0,05 quiere decir, que es el salto que está más tiene relación con el TMC.

\begin{tabular}{|l|l|r|r|r|}
\hline & \multicolumn{1}{|c|}{ ABK } & \multicolumn{1}{c|}{ SJ } & \multicolumn{1}{c|}{ CMJ } \\
\hline $\begin{array}{l}\text { Test } \\
\text { Motor } \\
\text { complejo } \\
\text { de }\end{array}$ & $\begin{array}{l}\text { Correlación } \\
\text { de Pearson }\end{array}$ & $-0,456$ & $-0,245$ & $-0,349$ \\
\cline { 2 - 5 } Caminero & $\begin{array}{l}\text { Sig. } \\
\text { (bilateral) }\end{array}$ & 0,087 & 0,379 & 0,202 \\
\cline { 2 - 5 } & N & 15 & 15 & 15 \\
\hline
\end{tabular}

Tabla 3. Correlación TMC - Indicadores DISCUSIÓN

En la presente investigación se buscó establecer la relación existente entre las capacidades coordinativas y la potencia en nadadoras juveniles de la Ciudad de Tunja. Al aplicar cada uno de los test y hacer el análisis de los mismos se observó que no existe correlación entre las variables; Test de Bosco y el Test Motor Complejo de Flaviano Lorenzo Caminero, en la tabla 3 se resalta que el más cercano a tener relación fue el despegue en ABK con valores de significancia de 0,087 el cual fue el más cercano a estar por debajo de 0,05 . Así como, los resultados evidenciados en el estudio de (Moreno, T., 2016) donde se evaluaron las capacidades coordinativas con el TMC y la capacidad de fuerza explosiva mediante el test de salto vertical y lanzamiento de balón. Los resultados obtenidos entre las variables muestran que no existe correlación en ninguno de los dos sexos. Entre las variables capacidad coordinativa y lanzamiento de balón en niños se observa una correlación baja de $(p=0.0031$; $p<0.05 ; r=0.3912)$. En niñas no hay correlación de estas variables.

Por otra parte, los resultados obtenidos en el TMC, muestran que el menor tiempo fue de 23,82 sg, que comparado con los resultados del pre-test del grupo experimental, en el estudio realizado por (Parada \& Agudelo, 2018) la media es de $35,8240 \mathrm{sg}$, que quiere decir que a la hora de aplicar el test las nadadoras presentan una 
mejor condición de las capacidades coordinativas con respecto a los tenistas.

En cuanto a la potencia se puede observar en la investigación realizada por (Gamardo, 2017), donde si hubo correlación entre la potencia y la composición corporal en niños de 6 a 13 años, que la media para el despegue en $A B K$ es de $21,8 \mathrm{~cm}$ y para $C M 17,64 \mathrm{~cm}$, valores inferiores a los obtenidos en el grupo de nadadoras donde la media para ABK es de $25,173 \mathrm{~cm}$ y CMJ $22,508 \mathrm{~cm}$, posiblemente dichos resultados se dan por la diferencia de edad entre los grupos participantes.

En la investigación de (Tejada, 2013), en la cual hubo correlación directa $(r=0,7)$ y $r=0,6)$ entre la velocidad de las jugadoras y el salto, teniendo en cuenta, los resultados de las mujeres se observa que la media para el despegue en $A B K$ es de $40,7 \mathrm{~cm}$ un valor superior al obtenido en la presente investigación, al ubicar estos resultados en la tabla de percentiles de (Jáuregui \& Ordóñez, 1993) (altura del salto vertical Abalakov para hombres y mujeres Colombianos) se evidencia que las nadadoras se encuentran en el percentil 40, que es muy bajo respecto a las jugadoras de Ultimate Fresbee, que se encuentran en el percentil 100. Se puede deducir que los resultados se dan por las características del deporte, edad y años de experiencia deportiva.

En el estudio descriptivo transversal realizado por (Luarte \& González, 2014), donde se aplicaron los tres saltos utilizados en la presente investigación, se halló que si hay diferencias significativas en cuanto al test de salto en CMJ $(28,50 \mathrm{~cm})$ y ABK $(32,90 \mathrm{~cm})$ en relación a la posición de juego de las voleibolistas, en el SJ $(23,08 \mathrm{~cm})$ no se hallan diferencias significativas en función de la posición de juego, se encuentra que son valores mayores a los resultados de las nadadoras, dado a que el salto vertical en el voleibol es fundamental para ejecutar todas las acciones impuestas por el juego; principalmente en el remate y en el bloque, una de las características de este deporte es la fuerza explosiva y fuerza elástico explosiva que se traduce en potencia y que tiene un papel determinante en el tren inferior.

\section{REFERENCIAS BIBLIOGRÁFICAS}

Bosco, C. (1982). A simple method for measurement of mechanical power in jumping. Appl Physiol.

Caminero, L. (2009). Diseño y estudio científico para la validación de un test Motor original, que mida la coordinación motriz en alumnos/as de educación secundaria obligatoria. España: Universidad de Granada.

Gamardo, P. (2017). Potencia muscular de niños entre 6 y 13 años de edad de las Escuelas deportivas del instituto Pedagógico de Caracas, Venezuela: Universidad Pedagógica Experimental Libertador-Instituto Pedagógico de Caracas.

González, G. (2009). Capacidades Coordinativas. Deporte6am. 
Jáuregui, G., \& Ordóñez, O. (1993). Aptitud física: pruebas estandarizadas en Colombiaa: Ministerio de Educación Nacional. Bogotá, D.C..

Luarte, C., \& González, M. (2014). Evaluación de la fuerza de salto vertical en voleibol femenino en relación a la posición de juego. Ciencias de la Actividad Física UCM., 43-52.

Meinel, K. (2004). Teoría del movimiento. Sintesis de una teoría de la Motricidad deportiva bajo el aspecto pedagógico . Buenos Aires:Stadium

Moreno, T. (2016). Correlación entre fuerza explosiva y capacidades coordinativas en escolares del Liceo León de Greiff de Tunja. Colombia: Universidad Pedagógica y Tecnológica de Colombia.

Parada, M. (2018). Efecto de entrenar por modelamiento para el desarrollo coordinativo en tenistas de 10-16 años. Colombia: Universidad Pedagógica y Tecnológica Colombia.

Tejada, C. (2013). Correlación entre la potencia en miembros inferiores(altura de despegue del salto) medida on el test de Bosco y la velocidad frecuencial(medida con el test de 30 y 60 metros planos)de la selección Colombiana de ultimete fresbee. Viref, 147-162. 